\title{
Annichilare o annichilire?
}

\author{
Vittorio Coletti
}

PUBBLICATO: 13 DICEMBRE 2019

\section{Quesito:}

Alcuni lettori ci pongono domande su quale, tra annichilire e annichilare, debba considerarsi forma "corretta"; un lettore in particolare formula la richiesta in riferimento all'ambito della fisica teorica; un altro chiede se ci sia una differenza semantica tra le due forme verbali. Un lettore ha invece dubbi sulla flessione del verbo annichilire: annichila o annichilisce? Un altro infine chiede quale sia, specie in ambito filosofico, il significato di annichilazione.

\section{Annichilare o annichilire?}

A

nnichilire e annichilare sono due sinonimi sostanzialmente perfetti ('ridurre a nulla, annientare, annullare, azzerare', anche in senso figurato), cosa che non sempre succede con varianti di coniugazione di uno stesso verbo (colorire/colorare, arrossire/arrossare), in cui qualche differenza di significato è spesso più o meno nettamente percepibile. Anche se sono antichi sostanzialmente allo stesso modo, annichilare lo è un po' di più ed era più attestato in passato; deriva dal latino della scolastica e della vulgata di S. Gerolamo: ad + nichil (nel latino medievale $h$ era pronunciata $/ \mathrm{k} /$ ) +-are, poi circolato quasi sempre in ambito filosofico e soprattutto mistico-religioso (Ugo Panziera: "il dilecto del fuoco celestiale annicchillava la pena del fuoco materiale"), e più tardi anche scientifico (Galileo: "il moto continuamente scemando... finalmente si annichila") e poi genericamente colto (Cesare Beccaria: "La mano risoluta del legislatore deve annichilare si fatte imposture"), come attestano queste citazioni dal GDLI. Anche la variante in -ire è attestata già nel Medioevo, specie nel significato mistico-religioso, ma è meno frequente (nell'OVI ha una sola occorrenza contro 4 in -are) e (stante il TLIO) si trova soprattutto in autori non toscani. Dalla forma originaria in -are sono derivati già anticamente annichilamento, annichilazione e annichilanza, sinonimi (il terzo del tutto scomparso) nel senso di 'annientamento, umiliazione radicale, riduzione (in filosofia) al non essere, al nulla', ecc. Annichilimento (da annichilire) è venuto dopo (il corpus medievale dell'OVI non lo registra e il GDLI lo attesta solo da fine Ottocento) e manca ancora nel TommaseoBellini; ma ha lo stesso significato dei sostantivi derivati dalla forma in -are.

Va fatta una breve riflessione su questi cambi di coniugazione. I più significativi si sono verificati nel passaggio dal latino classico a quello volgare e quindi al toscano (da ridére a ridere, da florére a fiorire, da cápere a capire, da trémere a tremáre ecc.) e prevalentemente da -ere a-ire o da-ere a -are. Più rari e di epoca ormai volgare i metaplasmi da coniugazione in -are (la più attiva da sempre) a quella in -ire. Allo stato delle conoscenze risulta che la nascita delle due varianti o è quasi coeva (ammansare e ammansire sono entrambi del XIV secolo, così assordare e assordire) oppure quella in -ire è più tarda (rattristare del XV e rattristire del XIX). Interessante il caso di suffissati da una base aggettivale che danno -are (curiosare, pazientare) ma, se parasintetici (cioè anche con prefisso), -ire (incuriosire, spazientire più recenti dei parenti in -are). Mentre i metaplasmi dal latino sono fenomeni evolutivi, nel senso che una forma sostituisce l'altra, questi interni all'italiano sono fenomeni giustappositivi, una forma non esclude necessariamente l'altra e le due possono convivere come sinonimi (come i verbi qui in esame) oppure si specializzano ognuna con un proprio significato (impazzare e impazzire, attestati entrambi dal XIII secolo). 
Posto dunque che entrambi i verbi (annichilare e annichilire) sono corretti e ammissibili, tutto farebbe pensare che quello in -are (con la sua morfologia) sia il più comune e consigliabile per ricchezza di storia. Sennonché uno sguardo al GDLI e soprattutto al Primo Tesoro (PTLLIN) di romanzi novecenteschi ci mostra un singolare capovolgimento della situazione: niente annichilare e derivati nei romanzi del Premio Strega e solo annichilire (con la sua morfologia in -isc-al presente) e derivati (annichilimento). Il GRADIT classifica annichilare di Basso Uso e annichilire COmune; il Vocabolario Treccani ant. annichilare e comune annichilire. Il Sabatini-Coletti registra senza riserve annichilire e classifica come non com(une) annichilare e cosi fa lo Zingarelli. Annichilimento (che il GDLI, come si è detto, attesta solo da fine Ottocento) precede nei lemmari (di Sabatini-Coletti e Devoto-Oli) la variante annichilamento (ancora comune in Leopardi), oppure questa è classificata rara (Zingarelli) o di basso uso (GRADIT). Nei Promessi sposi "la misera ... era annichilata", ma in Moravia il narratore "rimase... annichilito". È vero che Gadda (ma è Gadda!) adopera ancora annichilandola, ma è l'uso più recente del verbo attestato dal GDLI, perché tutte le citazioni più moderne nello stesso dizionario sono invece da annichilire (Bacchelli, Palazzeschi, Pratolini, Umberto Fracchia). Il Vocabolario della poesia italiana del Novecento (Zanichelli, 1995) attesta solo un annichiliti (in Ungaretti).

Google libri ci consente di cogliere i primi indizi del recupero di posizioni della variante in -ire da fine Settecento. Nella seconda metà dell'Ottocento, se il Tommaseo-Bellini registra senza restrizioni annichilare e dà come vitando annichilire, su cui sentenzia: "non è né com., né proprio, né necessario, né bello", il Petrocchi (Nóvo dizionario universale della lingua italiana, Treves,I892) comincia a pareggiare le due varianti. Oggi quella in -ire sembra voler scalzare la forma madre in -are (che pure resiste ancora abbastanza), tanto che su Google per I8.ooo occorrenze di annichilare ce ne sono ben 99.800 di annichilire e l'archivio di "Repubblica" (dal 1984) dà solo 3 casi di annichilare contro 358 di annichilire, 366 di annichilisce contro I3 di annichila.

I numeri sono eloquenti. Anche se la forma in -are è tutt'altro che scorretta (anzi è etimologicamente quella originaria), oggi è comune e quindi più consigliabile quella in -ire. Lo stesso si dica per l'opzione tra annichilamento e annichilimento. L'uso fa la regola.

Quanto ad annichilazione (documentato già nel Trecento: OVI, TLIO), è considerato nel GRADIT sinonimo di annichilimento, ma ha anche significati specifici nel linguaggio della teologia ("pena prevista per i malvagi consistente nell'annientamento dell'anima dopo la morte" e anche "subordinazione e smarrimento della propria personalità che si realizza nell'unione con Dio") e in quello della fisica ("interazione tra una particella e la sua antiparticella con trasformazione integrale della loro massa in energia"), cosi come del resto annichilimento ha (o ha avuto) un significato specifico in psichiatria ("tecnica particolare della terapia con elettroshock che determina uno stato amnestico confusionale").

\author{
Cita come: \\ Vittorio Coletti, Annichilare o annichilire?, "Italiano digitale", 2019, XI, 2019/4 (ottobre-dicembre) \\ DOI: 10.35948/2532-9006/2020.3245 \\ Copyright 2019 Accademia della Crusca \\ Pubblicato con licenza creative commons CC BY-NC-ND
}

